\title{
High-resolution ERP mapping of cortical activation related to implicit object-location memory
}

\author{
Jonathan S. Murphy * Ciara E. Wynne, Edel M. O’Rourke, Seán Commins, Richard A.P. Roche \\ Dept. of Psychology, National University of Ireland, Maynooth, Co. Kildare, Ireland
}

\section{A R T I C L E I N F O}

\section{Article history:}

Received 22 October 2008

Accepted 7 August 2009

Available online 14 August 2009

\section{Keywords:}

Implicit memory

Spatial ability

ERP

P300

Gender

\begin{abstract}
A B S T R A C T
High-density event-related potentials (ERPs) were recorded during an object recognition task which involved task-irrelevant changes in the location of studied objects. Participants categorised objects as studied or novel while data were analysed to ascertain the effect of the location changes on performance and waveform topography. Our results indicate that humans can classify objects faster and more accurately when using implicit spatial memory. Individual differences observed in object recognition proficiency were absent if objects were presented in their 'correct' location. In a second experiment we replicated the behavioural findings while manipulating viewpoint to discount scene recognition as an underlying factor. We propose a model which includes activation of the right medial temporal lobe prior to P300 elicitation to account for the prophylactic effect of implicit processing on object recognition. Hemispheric differences in parietal componentry dependant on sex of participant were also observed and are discussed in relation to differential strategies.
\end{abstract}

(c) 2009 Elsevier B.V. All rights reserved.

\section{Introduction}

Humans cannot navigate successfully in the environment without the basic capacity to remember the location of objects. Object-location memory depends on a variety of component processes such as object processing, spatial-location processing or memory for the locations of individual items and memory for occupied locations (Puglisi et al., 1985). Like other types of memory processes, encoding and recognition of object-location information can occur consciously or unconsciously (Caldwell and Masson, 2001; Hasher and Zacks, 1979). According to the dual-process theory (Aggleton and Brown, 1999; Rugg et al., 1998), recognition of object-location is supported by two kinds of memory: recollection and familiarity. Recollection like explicit memory refers to the consciously controlled retrieval of information from a prior study episode. In contrast, familiarity or implicit recognition is the phenomenological experience that a particular item reminds one of something (Jacoby and Kelley, 1992).

Jacoby (1991) developed the process-dissociation procedure to assess the independent effects of explicit and implicit memory within a single task. The basis of this procedure was to set responses based on recollection and familiarity against each other to provide better measures of conscious and unconscious processes. Using this procedure Caldwell and Masson (2001) discovered that only the implicit learning and retrieval of object-

\footnotetext{
* Corresponding author. Tel.: +3531 108 6086; fax: +3531 1084767 . E-mail address: jonathan.s.murphy@nuim.ie (J.S. Murphy).
}

locations is influenced by the strength of a habit (practice) with object-locations. They found that habit influences unconscious object-location memory in two important ways. First, when an object is placed in its usual location on a particular episode, habit supports the performance of accurately locating that object. Second, trying to find an object that has been placed in an unusual location can be hampered by a strong habit that associates that object with a different location. This latter influence of habit is a form of memory slip and is particularly likely to occur when strong habits are involved (Hay and Jacoby, 1996). It has been proposed that this habit is sexually dimorphic and stronger in females (Eals and Silverman, 1994).

Some studies investigating implicit memory for location have reported sex differences evident in performance (Alexander et al., 2002; Cherney and Ryalls, 1999; Eals and Silverman, 1994; Turner, 1993). These sex differences manifest in females' superior performance and also appear to have a hemispheric dissociation where left hemispheric activation is greater in females. The reported higher female scores and hemispheric bias has been suggested to result from differential female/male strategies being used (Turner, 1993). Evidence from studies in adults shows that disrupting the phonological loop, which holds information in working memory using inner speech (Baddeley, 1990), with a concurrent verbal task (articulatory suppression), interferes with the 'object-to-position assignment process', suggesting that it relies to some extent upon verbal coding (Postma and De Haan, 1996). Alexander et al. (2002) hypothesise that females may be more likely than males to show better performance in the right hemispace on some spatial tasks because of a relatively enhanced 
efficiency of categorical spatial processing in the left hemisphere. Additionally, Bowers et al. (1998) has suggested females use verbal mediation to complete spatial tasks. This additional left hemispheric activation may account for females' superior performance for right hemispace stimuli. Notably, the female advantage in location memory also occurs when no instruction to attend to object-locations is given (Bowers et al., 1998).

Since spatial memory plays an important role in everyday life, remembering the location of objects within the environment can be a process which is mainly or fully automatic/implicit (Hasher and Zacks, 1979). Richardson and Spivey (2000) investigated whether spatial information is encoded automatically. Participants were presented with items at one of four possible regions on a computer screen, along with unrelated facts presented aurally. Then, while looking at a blank grid, participants heard a true or false statement relating to one of the four facts, and replied true or false. Even though location was irrelevant to the task, it was found that under certain conditions during recall, participants made significantly more saccades to the empty region of space where semantic information had previously been presented. In another study, Ryan et al. (2000) manipulated object-locations in a scene recognition task and consequently found dissociations between memory effects that participants explicitly reported and the implicit memory that their eye movements revealed. More recently Van Asselen et al. (2005) studied the influence of incidental (automatic) and intentional (automatic and effortful) learning conditions on route learning and found intentional learning proved superior on navigational tasks but that there was no significant difference in performance between conditions on subsequent landmark-recognition and landmark-ordering tasks suggesting that object-location memory can be encoded implicitly.

Our understanding of the structures underlying object-location recognition has been advanced through lesioning studies. Introduction of novel objects or indeed a rearrangement of the spatial orientation of the objects already present in an array produces a renewal of exploration in animals (Commins et al., 2003). Animal lesion studies have highlighted the different contributions of hippocampal and parahippocampal structures to this exploratory behaviour. Hippocampal-lesioned animals increase their reactivity to a novel object (Save et al., 1992), entorhinal-lesioned animals fail to react to a novel object (Galani et al., 1998; Mumby and Pinel, 1994) and perirhinal-lesioned animals show impairments in object recognition tests (Ennaceur et al., 1997). Hippocampal-lesioned animals also fail to increase their reactivity following a spatial change of objects already present in the environment (Galani et al., 1998). This does not seem to be the case for perirhinal-lesioned animals (Steckler et al., 1998). This last point highlights a dissociation in hippocampal and parahippocampal structures for spatial and non-spatial processing. Human lesion-patient studies have demonstrated the effect of medial temporal lobe lesions on the recall of locations of objects in an array, finding a right lateralisation in the hippocampus for the processing of objectlocation (Crane et al., 1995; Smith and Milner, 1981, 1984, 1989). Evidence for this hemispheric asymmetry also comes from imaging studies. Milner et al. (1997) report two PET experiments in normal volunteer subjects, where activation was observed consistently in the right anterior parahippocampal gyrus during retrieval of object-locations.

Wang et al. (2002) demonstrate that object-locations may be implicitly encoded relative to their surroundings. Despite instruction to learn and retrieve object-location associations using landmarks in a spatial array, participants performed successfully in a 'fixed-no cue' condition where the landmarks were not present. The experimenters concluded from reaction times and eye movement traces during the object-location retrieval tasks that encoding using a fixed external reference frame (screen-based representations of object-locations) is equivalent to the encoding of fixed-landmark to object-location representations. The results indicate that a screen-based spatial representation may be implicitly encoded and stored and perhaps this representation may even be adopted to perform the fixed-landmark condition. In addition, during the encoding phase subjects were found to move their eyes back and forth between the currently presented object and the location of the previously displayed object suggesting that some form of object-based spatial relationships may also be encoded directly and quite early. Reaction time data and eye movement traces indicate that multiple forms of representations may be used to encode and retrieve object-locations.

Spatial relationships among objects have been shown to be important for object recognition in complex scenes (Chun and Marois, 2002; Ullman, 1996). Implicit spatial memory has been studied with regard to perceptual object recognition, i.e. the contextual cuing paradigm (Chun and Jiang, 1998). Here, participants were required to perform a visual search task in which the spatial configuration of the array elements (a target ' $T$ ' and rotated ' $\mathrm{L}$ ' distractors) was random or repeated across the experiment. Participants were found to respond faster to targets in repeated than in new configurations of the spatial array (e.g., Chun and Jiang, 1998; Chun, 2000; Olson and Chun, 2002). It is suggested that participants learn the invariance between the locations of array elements and the target location in the repeated configurations.

The aim of the current study is to assess, both behaviourally and electrophysiologically, the effect of implicit spatial memory on object recognition and identify sources that may underpin such memory. To date there has been little work investigating the electrophysiological biomarkers of implicit spatial memory. With this in mind we also intend to investigate the electrophysiological underpinnings of the sex differences reported in the literature. We report the results of two experiments. The study utilizes the computerised 'Spatial Grid Task' based on the Milner paradigm (e.g., Johnsrude et al., 1999; Milner et al., 1997; Owen et al., 1996) to test participants' object recognition memory explicitly, while simultaneously testing implicit memory effects of object-location. Objects were encoded one at a time in a three-dimensional spatial grid, so that participants never saw the complete array, allowing for greater manipulation of conditions and processing demands at retrieval. All objects were presented in colour with 3D rendering, and encoded and retrieved from a stationary, oblique viewpoint which permitted an egocentric (person-centred) frame of reference. In the test phase, a studied or 'target' object is presented in its 'correct' (i.e. previous) location. Additionally, novel or 'distractor' objects were also presented in one of four random locations (i.e. neither a familiar object nor location). In Experiment 1 highdensity, 128-channel EEG recordings were used to investigate the scalp waveform componentry and electrical dipole sources associated with object-location memory. ERP data were analysed and dipole source localisation carried out using Brain Electrical Source Analysis (BESA@) software. Experiment 2 was a behavioural experiment, utilising the same Spatial Grid task but with the addition of two new viewpoints from which participants had to respond in the test phase.

It is predicted that upon encoding object information participants will implicitly record the object-locations due to the influence of habit (Caldwell and Masson, 2001) and that this influence will have a beneficial effect on performance. Thus, it is hypothesised that response latency will be faster and response accuracy will be greater upon presentation of target objects in their correct (i.e. previous) location than target objects in an incorrect location. It is also predicted that participants' performance will be similar from the altered viewpoints in Experiment 2 thereby eliminating scene recognition as a valid explanation for effects 
observed in Experiment 1. Specifically, in Experiment 1, we predict that retrieval should engage temporal and medial temporal lobe (MTL) areas (Roche et al., 2005). Consistent with previous studies of spatial memory (e.g. Owen et al., 1996; Johnsrude et al., 1999), it is predicted that waveforms will be lateralised in the MTL, predominantly in the right MTL. Cortically, we predict that the dorso-lateral prefrontal areas will be active for all trials (GoldmanRakic, 1987) in addition to the right posterior parietal lobe (Andersen et al., 1985). With regard to sexual dimorphism we predict superior performance in females and left hemispheric componentry over parietal areas additional to the male activation topography. This hemispheric difference should highlight the categorical and coordinate spatial strategies used by females and males respectively (Kosslyn et al., 1992). Finally we predict taskbased modulations of the amplitudes and/or latencies of ERP components associated with spatial processing, particularly increased parieto-occipital P300 for correct location presentations, consistent with the old/new findings of Mecklinger et al. (1997).

\section{Experiment 1}

\subsection{Method}

\subsubsection{Participants}

The participants in Experiment 1 consisted of an ad hoc sample of 20 undergraduate volunteers. The ages of the participants ranged from 20 to 27 years (mean age $=21.6$ years). Of these participants, 3 were removed for excessive EEG/EOG artifacts or head movements in their data or for misinterpreting the method of responding. Of the remaining 17 participants, 7 were females and 14 were right-handed ( 2 males and 1 female were left-handed). All participants had normal or corrected-to-normal vision and gave informed, written consent before participation. The experiment was conducted in accordance with the Code of Ethics of the World Medical Association and the ethical standards of the American Psychological Association (APA) as well as abiding by the NUI Maynooth University Ethics Code.

\subsubsection{Stimuli}

All stimuli were presented using E-Prime $\odot$ on an Intel Pentium 4 Processor (3.00 GHz CPU) and displayed on an LCD monitor. The task consisted of two computer-generated environments (Carpet and Grass) each with 8 different objects and two different landmarks. The objects and environments were constructed using Google SketchUp ${ }^{\mathrm{TM}}$, which allows for three-dimensional rendering in full colour. The 'environment' here refers to the background grid ( $4 \times 4$ equally spaced squares) on which the objects were presented. Two landmark objects were always present in each environment. Landmarks were used to allow an allocentric (object-centred) reference frame as well as the egocentric reference (grid) (see Johnsrude et al., 1999). All objects were presented in one of the 14 free squares in the grid. Participants' were subject to both environment tasks (grass and carpet) for control purposes in order to ensure that object-location memory was not subject to an environmental effect. Although environments and the objects they contain were different, the task required the same memory functions and as no significant differences were noted between environments, the behavioural paradigm will be described in reference to a single environmental task. All objects were of comparable size and none exceeded $15^{\circ}$ of visual angle.

\subsubsection{Procedure}

After the electrophysiological preparation (see next section for details), participants were seated $50 \mathrm{~cm}$ from the LCD computer screen on their own in a darkened, electrically shielded and sound-attenuated testing cubicle
$(150 \mathrm{~cm} \times 180 \mathrm{~cm})$ with access to a mouse for responses. A study block preceded a test block. Instructions were presented on screen prior to these blocks.

2.1.3.1. Study block. During the study block participants were asked to study the objects that appeared one at a time in the environmental grid with two stationary landmarks and were told that they would need to remember the objects for a subsequent recognition test. No reference was made to the location of the objects, only that the objects themselves had to be learned. The study block consisted of 64 trials of object presentations. Each of the 8 objects was presented in isolation 8 times in a pseudo-randomised order so that consecutive presentations of the same object did not coincide. The temporal sequence of a trial is displayed in Fig. 1. A fixation cross was presented first for $750 \mathrm{~ms}$, followed by the spatial grid with landmarks (e.g. lamp-post and water fountain) for $1500 \mathrm{~ms}$ and then the test stimulus was presented on the grid and remained onscreen for $2000 \mathrm{~ms}$. This cycle was repeated for the 64 trials. Stimulus presentations were marked on the EEG recording by transistor-transistor logic (TTL) triggers.

2.1.3.2. Test block. Following the 64 trials of object presentations, another set of instructions was provided. Participants were told to respond to previously studied (or 'old') objects that appeared during the test block by pressing the left mouse button with their index finger. If a 'new' object (i.e. not shown in the study phase) was presented, then the right mouse button should be pressed with their middle finger. For the Test block, the sequence of a single trial followed the same pattern as above (Fig. 1), with the stimulus duration as the response interval, $<2000 \mathrm{~ms}$. This trial sequence was repeated for 128 trials of object presentations. Three test conditions were constructed using either the 8 'old' (studied) objects or 8 'new' (distractor) objects and were presented in a pseudo-randomised order, to test the implicit learning of object-locations. The first condition (Target Object condition) involved the presentation of each of the 8 'old' objects in their previously studied or 'correct' location a total of 4 times each ( $n=32$ trials). The second condition (Target Object-Incorrect Location condition) involved the presentation of each of the 8 'old' objects in 4 allocated 'incorrect' locations ( $n=32$ trials). The third condition (Distractor condition) then presented 8 novel or distractor objects in 4 random locations that were unfamiliar to the participant; these 32 trials were each repeated twice ( $n=64$ trials).

Accuracy and reaction times were both recorded during the test phase of the experiment. A correct response occurred if the participant pressed the left mouse button when an 'old' object appeared and the right mouse button when a 'new' object appeared. Pressing the opposite button than required or failure to respond within $2000 \mathrm{~ms}$ resulted in an incorrect response. Reaction times were measured as the interval between presentation of the stimulus and the response, and were recorded for both correct and incorrect trials. TTL triggers were again used to record stimulus presentations and responses on the continuous EEG.

2.1.3.3. Electrophysiological recording and setup. EEG activity was recorded with tin electrodes (BrainVision $\odot$ ) mounted in an elastic cap fastened with a chest strap (Easy-Cap $\odot$ ). Data were collected from 128 scalp sites, using the extended version of the International 10-20 system for electrode placement (American Electrophysiological Association, 1999). The reference electrode was located on the nasion at the tip of the nose. Vertical and horizontal eye movements were recorded using electrooculography (EOG). VEOG was recorded from electrodes located above and below the left eye, and HEOG from electrodes at the outer canthus of each eye. Blinks were averaged off-line and a blink reduction algorithm was applied to the data. This algorithm involved automatic artifact correction employing variations of the Berg and Scherg (1991), and Ille et al. (2002) strategies. The impedance level was kept to below $10 \mathrm{k} \Omega$ in all cases. The amplifier used was supplied by BrainVision $\odot$. EEG activity was amplified using a band-pass of $0.16-100 \mathrm{~Hz}$ and a gain of 1000 . The conversion rate was $2000 \mathrm{~Hz}$ per channel and the range was $150 \mathrm{mV}$.

\subsubsection{Data analysis}

2.1.4.1. Behavioural data. Response accuracy was calculated automatically by E-Prime $\odot$ and manually collated into accuracy totals for each response (correct,

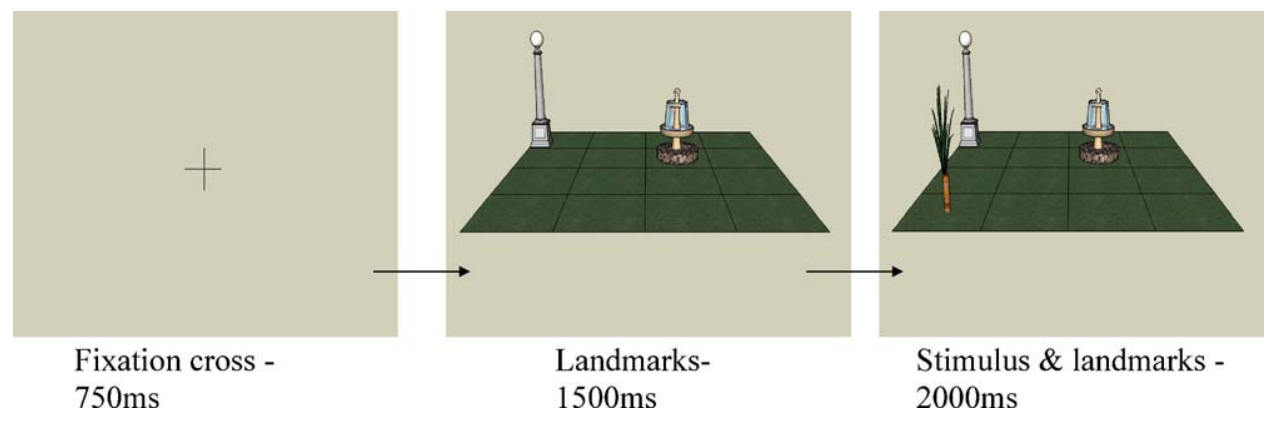

Fig. 1. Temporal sequence of a single trial in the experiment. 
incorrect) and condition (Target Object, Target Object-Incorrect Location, Distractor condition). All latencies were also calculated automatically by E-Prime $\odot$ and grouped as described above. Post hoc paired-sample $t$-tests were Bonferroni corrected. A median split of the data was conducted based on reaction times to compare differences between participants whose performances were affected by the location of the studied objects and those that performed at a similar level regardless of object-location. This resulted in two groups of participants, one with a small effect of object-location on reaction time (Good Recognition Group) and one with a large effect (Poor Recognition Group). Eight participants, from either side of the median, were included in each group. The Good Recognition Group had 4 males and 4 females and the Poor Recognition Group consisted of 5 males and 3 females, leaving one male participant, i.e. the median, excluded from both groups.

2.1.4.2. ERP data. Recordings were notch filtered off-line at $50 \mathrm{~Hz}$. EEG data were digitized at a sampling rate of $500 \mathrm{~Hz}$, and were averaged off-line using BESA৫ software. Epochs that exceeded $50 \mu \mathrm{V}$ were discarded from the analysis. The $100 \mathrm{~ms}$ pre-stimulus interval was used for baseline correction. Stimulus-locked average ERPs time-locked to the onset of the landmark presentations and test objects were computed for each participant with epochs of $-100 \mathrm{~ms}$ to $1200 \mathrm{~ms}$ at all electrode sites. Participant EEG was used to create 6 separate conditional ERPs: (1) landmark presentation during study; (2) landmark presentation during test; (3) study object presentation; (4) target object presentation for correct responses; (5) target object-incorrect location for correct responses; and (6) distractor object presentation for correct responses.

Waveform component structure was assumed in an a priori manner with no prior knowledge of the pattern of effects. An overall grand-mean waveform was generated for each of the electrodes by collapsing across participants for each of the 6 conditional ERPs. From this, components of interest were identified through a visual analysis of the scalp data and differences between conditions were assessed at selected electrode sites using BESA@. From this initial inspection, possible comparisons were generated and were tested for statistical significance. Only scalp sites selected after a visual analysis of the data were included in the inferentia statistics (listed with the relevant results). Repeated-measures ANOVAs were conducted on mean and maximum peak latencies to assess amplitude and latency differences respectively for each of the identified components for all the comparisons. ERP differences were assessed for landmark presentations and stimulus presentations across the Study and Test blocks. In a second set of analyses, the effect of sex was assessed on the ERPs elicited by the experimental conditions. A mixed-factorial ANOVA was conducted on mean amplitudes recorded over the parietal cortex of the left (sites P1, P3, E118) and right (sites P2, P4, E121) hemisphere. Bonferroni post hoc tests were carried out as well as $t$-tests to ascertain specific differences. Statistical significance for all analyses was taken at ${ }^{*} p<0.05$ ${ }^{* *} p<0.01,{ }^{* * *} p<0.001$ ). Finally, in a third set of analyses a median split of the data was performed based on reaction times and differences in waveform topographies were tested for each of the stimulus-types.

2.1.4.3. Dipole source analysis. BESA@ employs a least squares fitting algorithm over which the user has interactive control. Source localisation proceeds by a search within the head model for a location where the sources can explain a maximal amount of variance (Scherg and Picton, 1991). A 4-shell ellipsoidal head model was used during dipole fitting in the current study. Whole-epoch modelling as well as individual component modelling was conducted using a data-driven step-wise approach and sequential fitting strategies where possible. Single dipoles were added to each model until the solution presented became physically implausible. Source waveforms were also plotted in BESA@ and model fit was assessed through residual variance (RV). MRI slices, also generated in BESA $\odot$ are included in the results but the modelled dipoles represent an oversimplification of the activity in the areas and should be considered as representative of centres of gravity of the observed activity rather than pinpoint localisations of exact generators (Molholm et al., 2004; Sehatpour et al., 2006). Using the inputted co-ordinates, the Talairach Daemon (Lancaster et al., 1997) identified the nearest grey matter structures.

\subsection{Results}

\subsubsection{Behavioural data}

As we found no significant difference between environment type (carpet and grass), the mean participant accuracy for both experimental environments were combined to give overall mean accuracy scores for each of the three stimulus conditions (target object, target object-incorrect location and distractor object condition). Overall, accuracy was high across the three conditions on both environments. Fig. 2a shows the percentage mean accuracy for each of these stimulus-types (Correct location targets: $97.6 \pm 0.79 \%$, Incorrect location targets: $94.8 \pm 1.1 \%$, and Distractors: $97.7 \pm 0.75 \%$ ). A repeated-measures ANOVA was carried out to compare the mean accuracy in the three conditions. The within-subjects effect revealed that there was a significant main effect for Accuracy across stimulus-types $[F(2,34)=3.281, p=0.05]$ but subsequent paired-samples $t$-tests found no significant differences between conditions. Reaction times are shown in Fig. $2 \mathrm{~b}$ and can be seen to be quicker on average for Correct location targets (737.8 $\pm 67.8 \mathrm{~ms})$ compared to Incorrect location targets $(788.2 \pm 88.4 \mathrm{~ms})$ and Distractors $(774.6 \pm 80.1 \mathrm{~ms})$. A
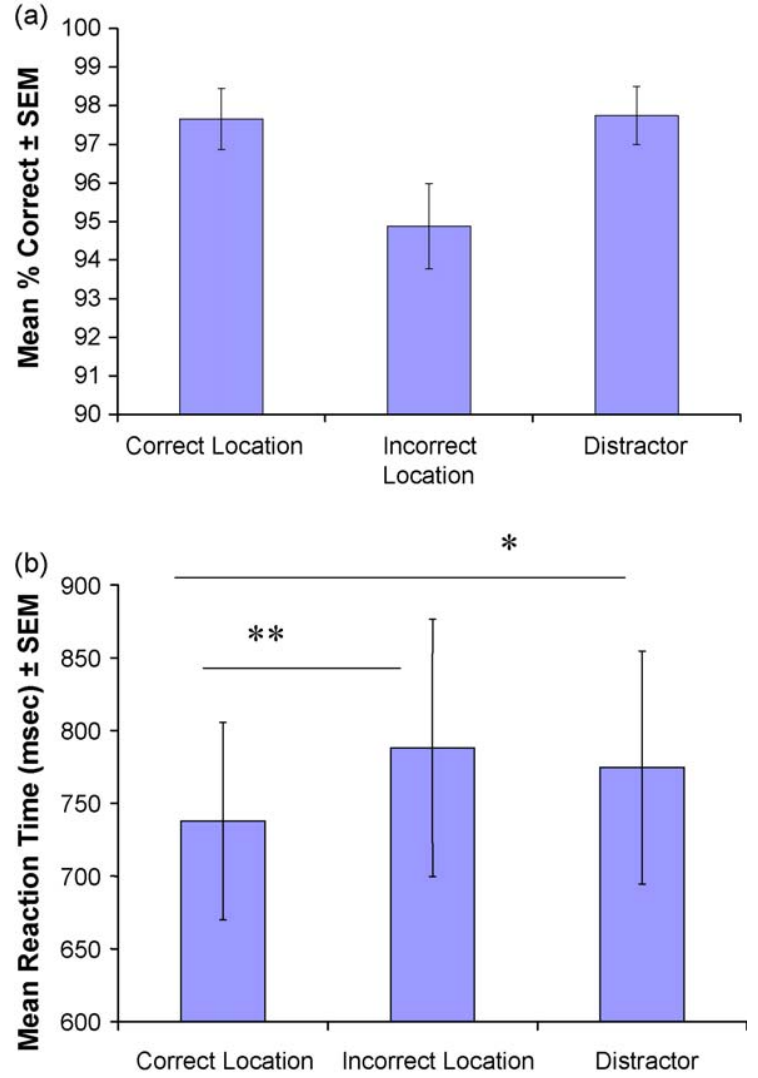

Fig. 2. (a) Mean Percentage Accuracy scores for each of the stimulus-type conditions. Error bars display Standard Error of the Mean. (b) Mean reaction times for each of the stimulus-type conditions. Error bars display Standard Error of the Mean. ${ }^{*} p<0.05,{ }^{* *} p<0.01$.

second repeated-measures ANOVA was carried out to compare mean reaction time in the three conditions. Reaction times were found to differ significantly with stimulustype $[F(2,36)=7.601, p=0.002]$. $t$-Tests found significant differences between Correct location targets and Incorrect location targets $[t(18)=4.250, p<0.005]$ and Correct location targets and Distractors $[t(18)=3.261, p<0.05]$ - Bonferroni corrected. A comparison of males' and females' performance revealed no significant differences in accuracy or reaction time.

An alternative analysis of the reaction time data was performed after a median split based on variance in reaction times for correctly and incorrectly located target objects (see Methods). The Good Recognition Group responded on average $17.59 \mathrm{~ms}$ slower to target objects incorrectly located vs. an average $87.67 \mathrm{~ms}$ delayed response for the Poor Recognition Group. Testing whether the performance difference between the groups was significant, paired-samples $t$-tests revealed no significant difference in reaction times across stimulus-types for the Good group whereas differences were found for the Poor group for Correctly vs. Incorrectly located targets $(p<0.001)$ and Correct location targets vs. Distractors $(p<0.01)$. Comparing reaction times between the groups using a mixed-factorial ANOVA (Stimulus-type $\times 3$ and Group $\times 2$ ), a significant effect of stimulus-type was found $[F(2,28)=10.206, p<0.001]$ as well as an interaction effect $[F(2,28)=4.699$, $p<0.05]$. The between-subjects variable 'group' also yielded a significant effect in the post hoc Bonferroni test $(p<0.05)$. A series of independent-samples $t$-tests were used to compare the Good Recognition and Poor Recognition Groups on each stimulus-type. No significant difference was found for Correct location targets (Good Group $M=712.91 \mathrm{~ms} S \mathrm{SD}=78.38$; Poor Group $M=771.9 \mathrm{~ms}$ SD $=35.89$ $t(14)=1.936, p>0.05)$ but significant differences existed for both Incorrect location targets $(M=730.5 \mathrm{~ms} \quad \mathrm{SD}=87.5 \quad$ vs. $M=859.57 \mathrm{~ms} \quad \mathrm{SD}=46.32$ $t(14)=3.687, \quad p<0.005)$ and Distractors $(M=738.9 \mathrm{~ms} \quad \mathrm{SD}=69.1 \quad$ vs. $M=816.07 \mathrm{~ms}$ SD $=64.25 t(14)=2.311, p<0.05)$ between Good Recognition and Poor Recognition Groups respectively.

\subsubsection{Event-related potentials}

2.2.2.1. Study vs. Correct location Test stimuli. Three waveform peaks were observed for Study stimuli, a P1, N2 and P3. Similar peaks were elicited for Test stimuli with additional post-P3 positivity (a P3b peak). The ERP waveforms for both the Study and Test stimuli across parietal sites resembled those recorded at $\mathrm{CPz}$ (displayed in Fig. 3a inset) where an extra late positivity was seen for the Test 
(a) $\mathrm{CPz}$

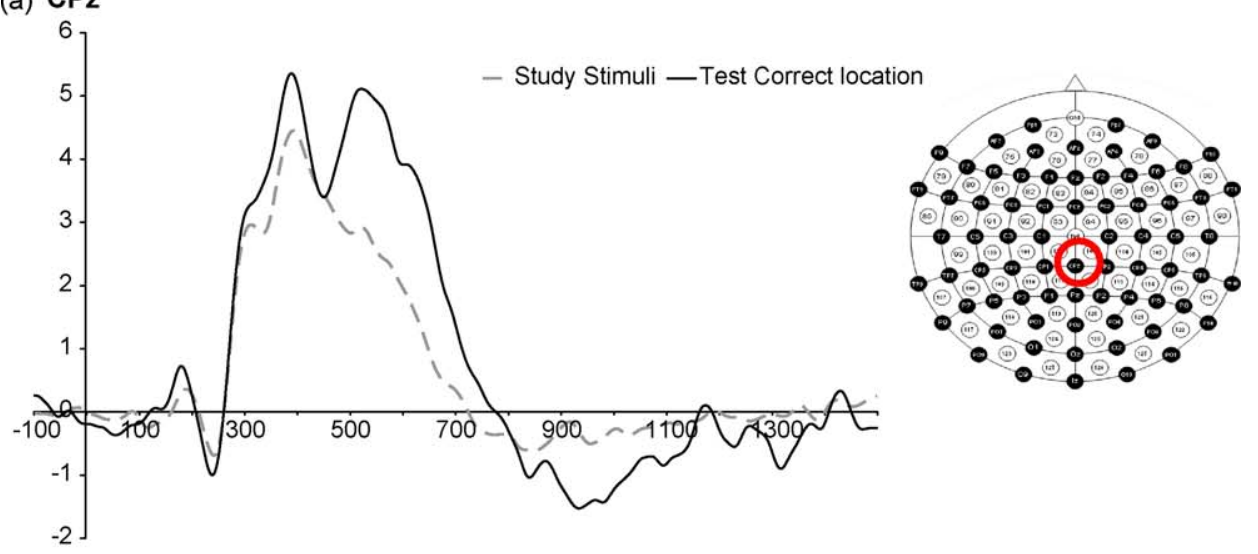

(b) $\mathrm{CPz}$
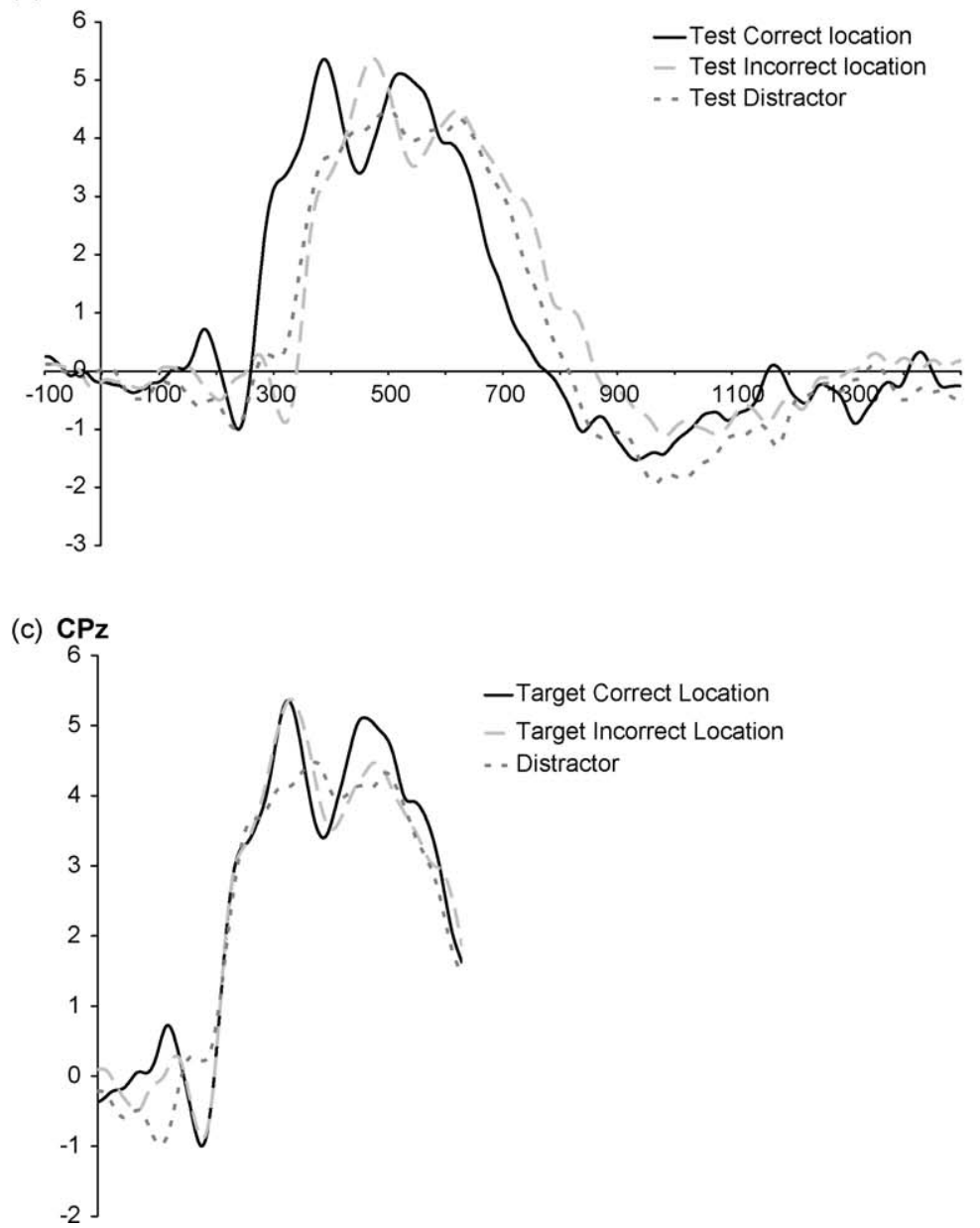

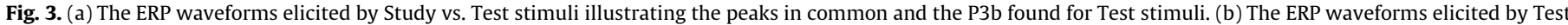

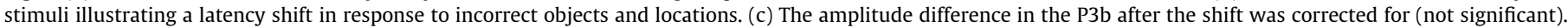

stimuli. To ascertain whether the waveforms differed significantly between 450$650 \mathrm{~ms}$, individual mean amplitudes were collated for both stimulus-types over this time interval and subjected to a paired-samples $t$-test. The average mean amplitude elicited by the Study stimuli was $2.45 \mu \mathrm{V}$ compared to $4.22 \mu \mathrm{V}$ for Correct location Test stimuli. A significant difference was found $[t(16)=4.681$, $p<0.0005$ ] indicating the presence of a P3b component uniquely elicited by Test stimuli.

2.2.2.2. Test stimulus comparisons. After examining differences between ERPs related to the study and test blocks, Grand Mean Average waveforms were generated for the different stimulus-types presented in the test block (Correct location targets, Incorrect location targets and Distractors). These waveforms are presented in Fig. 3b for site CPz. The latency difference seen here between correct location targets and the other stimuli was evident across parietal scalp sites (CP'XX' electrodes). Analyses were conducted on the latencies of individual maximum peaks calculated between 0 and $1000 \mathrm{~ms}$ from $\mathrm{CPz}$ (Mean latencies were $458.35 \mathrm{~ms}$, $525.41 \mathrm{~ms}, 519.41 \mathrm{~ms}$ for Correct location targets, Incorrect location targets and Distractors respectively). These maximum peaks represented individual P300s and the averages show a delayed response to objects presented out of their studied locations. A repeated-measures ANOVA yielded significant differences in waveform latencies $[F(2,32)=4.543, p=0.018]$ and a series of paired-samples $t$-tests showed this difference existed between Correct location targets and Incorrect location targets $[t(16)=2.607, p=0.019]$ and between Correct location targets and Distractors $[t(16)=2.438, p=0.027]$. 
Fig. 3c shows the waveforms after this latency difference has been removed. In order to correct for this difference and match the waveforms temporally, the 'Correct location targets' waveform was shifted $80 \mathrm{~ms}$ in time. This figure now allows an amplitude difference in the P3b to be seen. However, an ANOVA using mean peak data from the corresponding time intervals (480-580 ms for Correct location targets and 560-660 ms for the other stimuli), revealed that this difference was not significant $[F(2,32)=0.797, p>0.05]$.

The same latency difference can also be seen for the N2 as well as the P3, and appeared to be the consequent result of a P1 that was present for Correct location targets. An ANOVA testing differences in mean peaks over 150-200 ms was conducted and although a polarity difference can be seen in the average mean peaks relating to these stimuli, the ANOVA did not reach significance $[F(2,32)=2.109$, $p>0.05]$.

2.2.2.3. Male vs. female comparisons. For male/female comparisons ERPs were re-averaged so that even numbers of males (7) and females (7) were being compared. The selection of male subject data to be included was decided at random. Both groups included 6 right-handed participants. Amplitude differences between male and females were found across a number of midline and left parietal electrode sites. An example of this difference can be seen in Fig. 4 where conditional ERP waveforms from electrode $\mathrm{P} 3$ show the additional positivity recorded from female participants. A mixed-factorial ANOVA compared hemispheric mean amplitudes for males and females and found significant effects for stimulus-type $[F(3$, $120)=16.094, p<0.001]$. The effect of hemisphere just fell short of significance $[F(1,40)=4.053, p=0.051]$ but there was a significant interaction effect of sex $\times$ hemisphere $[F(1,40)=22.346, p<0.001]$ and the between-subjects effect (sex) was also significant $[F(1,40)=5.212, p<0.05]$. We conducted separate analyses on stimulus and landmark presentations. Using independent-samples $t$ test to compare male and female ERPs elicited by stimulus presentations, we found a significant difference in mean amplitudes over the left parietal cortex (P1, P3, E118) in the time interval $300-550 \mathrm{~ms}$ for the Study Stimuli $[t(40)=-3.550$, $p<0.001](M=2.40 \mu \mathrm{V}$ for males and $4.27 \mu \mathrm{V}$ for females $)$, Correct Location stimuli $[t(40)=-2.489, p<0.05](M=3.31 \mu \mathrm{V}$ for males and $4.83 \mu \mathrm{V}$ for females $)$, Incorrect Location stimuli $[t(40)=-3.506, p<0.001](M=2.48 \mu \mathrm{V}$ for males and $4.43 \mu \mathrm{V}$ for females $)$ and Distractors $[t(40)=4.669, p<0.001](M=2.23 \mu \mathrm{V}$ for males and $4.65 \mu \mathrm{V}$ for females. None of the comparisons between males and females for the right cluster (i.e. P2, P4, E121) reached significance $(p>0.05)$ nor did the $t$-test comparing mean amplitude responses to landmark presentations $[t(24)=1.950, p<0.05]$.

A further comparison of the electrophysiological data was undertaken for the time period identified (410-480 ms). Fig. 5 shows the hemispheric sex differences
ELECTRODE SITE P3
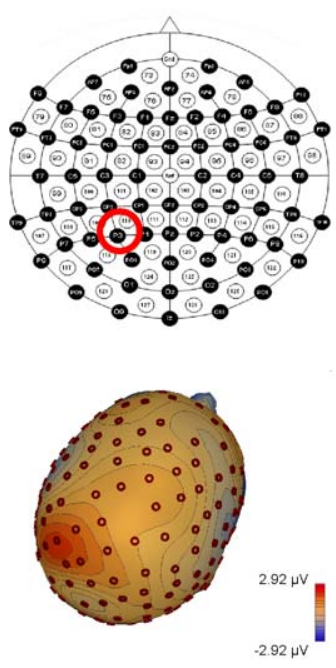

LANDMARKS

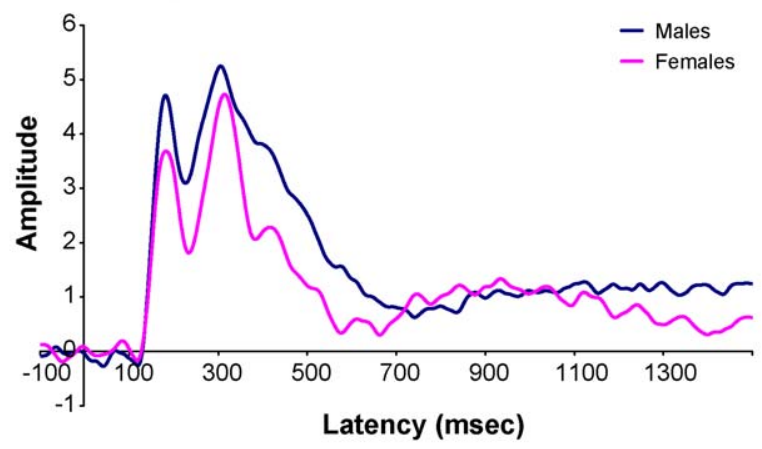

STUDY STIMULI

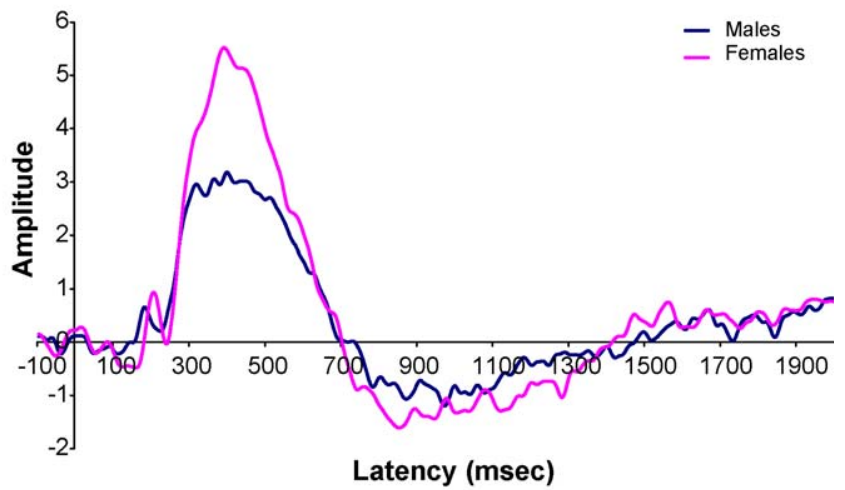

TEST STIMULI

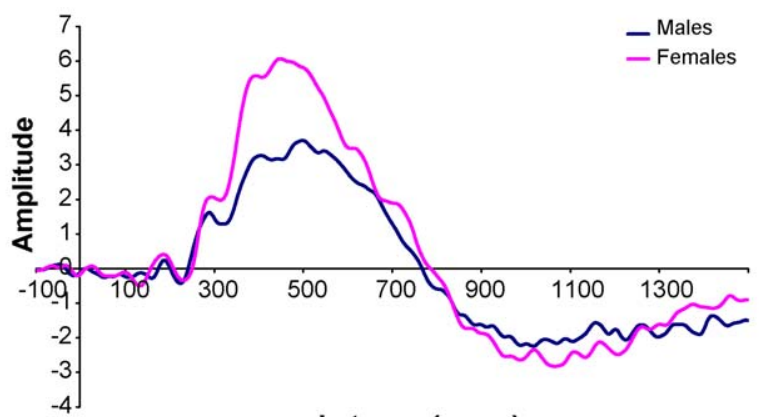

Latency (msec)

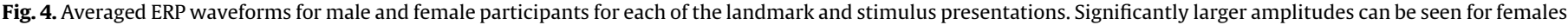

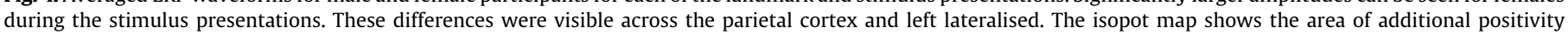
observed for females after the male activity had been subtracted out. 
FEMALE
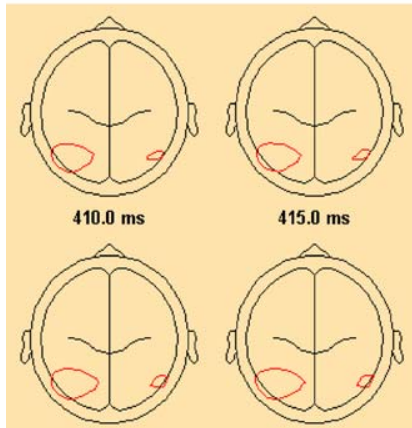

$415.0 \mathrm{~ms}$

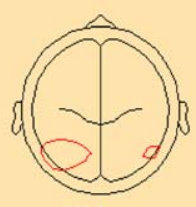

$425.0 \mathrm{~ms}$

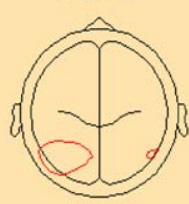

$440.0 \mathrm{~ms}$

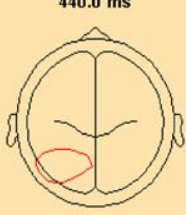

$455.0 \mathrm{~ms}$

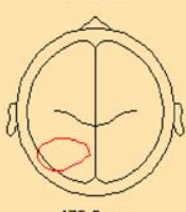

$470.0 \mathrm{~ms}$
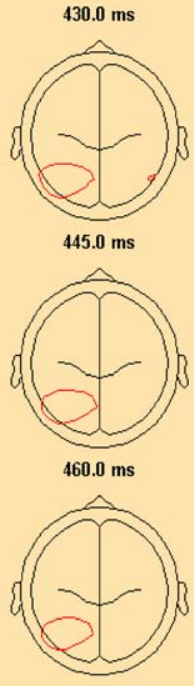
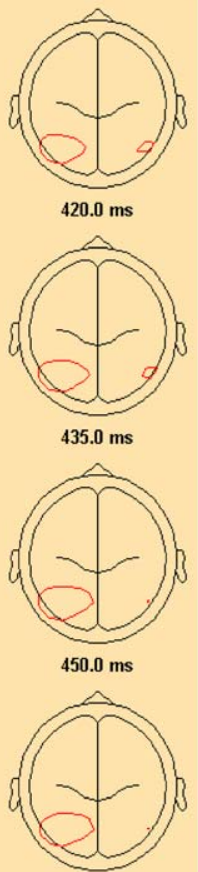

$465.0 \mathrm{~ms}$

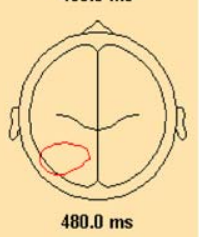

$445.0 \mathrm{~ms}$

EEG - C.S.D.

reference free $1.0 \mu \mathrm{V} / \mathrm{cm}^{2} / \mathrm{step}$

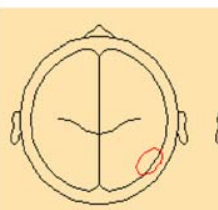

$410.0 \mathrm{~ms}$

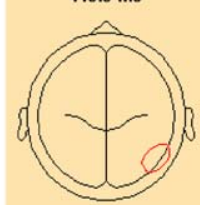

$425.0 \mathrm{~ms}$

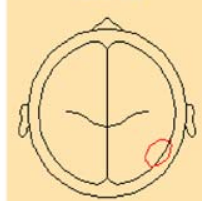

$440.0 \mathrm{~ms}$

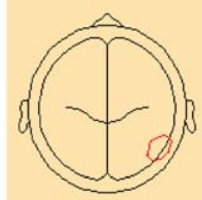

$455.0 \mathrm{~ms}$

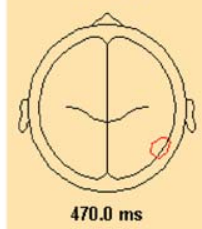

$470.0 \mathrm{~ms}$

$445.0 \mathrm{~ms}$

EEG - C.S.D.
MALE

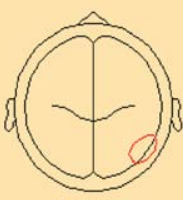

$415.0 \mathrm{~ms}$
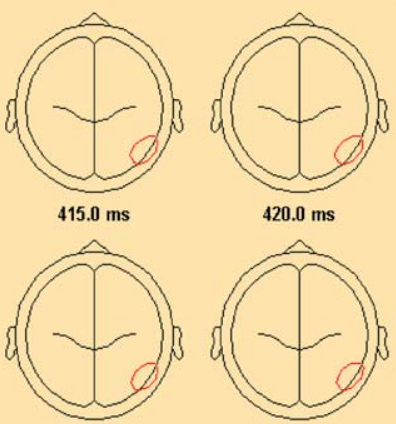

$430.0 \mathrm{~ms}$

$435.0 \mathrm{~ms}$
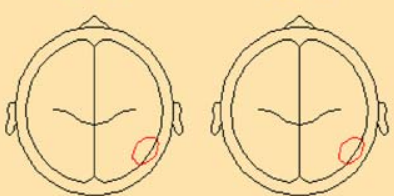

$445.0 \mathrm{~ms}$

$450.0 \mathrm{~ms}$
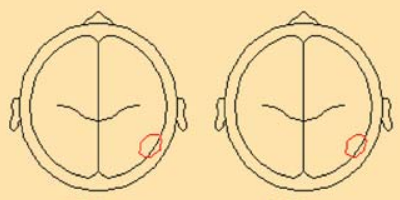

$460.0 \mathrm{~ms}$

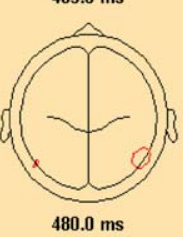

$475.0 \mathrm{~ms}$

reference free

$0.80 \mu \mathrm{V} / \mathrm{cm}^{2} /$ step

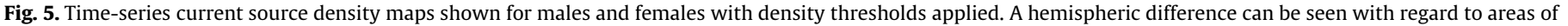
cortex with maximum current densities.

in high threshold time-series CSD (Current Source Density) maps. Current densities below $1.0 \mu \mathrm{V} / \mathrm{cm}^{2}$ cortex for females and $0.80 \mu \mathrm{V} / \mathrm{cm}^{2}$ cortex for males are filtered out, displaying only areas of highest density through the cortex across the time series demonstrating greater activity in the left hemisphere for females and the right hemisphere for males.

\subsubsection{Good Recognition Group vs. Poor Recognition Group}

Using the median split of the data based on reaction times (see above), waveforms elicited by each of the stimulus-types were calculated for both the Good Recognition Group and the Poor Recognition Group. The latency difference in the rise of the P300 existed in both groups. This was confirmed with a mixed-factorial ANOVA (Stimulus-type $\times 3$ and Group $\times 2$ ) where a main effect of stimulus-type was found $[F(2,28)=3.820, p<0.05]$ but the between-subjects 'group' variable had no significant effect on P300 max. peak latency. Amplitude differences in the P300 were observed between the groups and tested for each of the stimulus-types. Mean amplitudes were calculated for the time interval 300-500 ms for Study and Test stimuli for both groups. Independent-samples $t$-tests revealed significant differences in amplitudes between the groups for Incorrectly located objects and Distractors $(t(14)=2.824, p<0.05$ and $t(14)=-2.966, p<0.05$ respectively). Upon presentation of these stimuli, participants in the Poor Recognition Group showed significantly lower P300 amplitudes. Mean amplitudes for Correct location targets did not vary significantly between the groups. The relationship between reaction times and mean P300 amplitudes was investigated using Pearson product-moment correlation coefficient. There was a moderate negative correlation between the two variables $(r=-.431, n=48, p<0.005)$, with high amplitudes associated with lower reaction times.

\subsubsection{Dipole source analysis}

Models were generated from the Global Field elicited for the Study stimuli and each of the three stimulus-types from the test block. Table 1 shows the number of dipoles in each model, their Talairach co-ordinates and their approximate locations (supplied by the Talairach Daemon) and the Residual Variance (RV) of each model. Fig. 6a shows the dipole model for the Study stimuli. The dipole solution for the Test stimuli (Correct location targets) can be seen in Fig. 6b. The source waveforms shown illustrate each dipoles contribution to the models. Fig. $6 \mathrm{c}$ shows the dipole model for the localisation of the P1 identified for Correct location targets moving down through brain space on the $z$-axis. Readers are reminded however, that the P1 was only identified in terms of a polarity difference between the stimulus conditions and no significant amplitude differences were found. In localising the P1, a six dipole model consisting of bilateral frontal and parahippocampal dipoles (along with an occipital dipole and left temporal source) accounted for $\sim 90 \%$ of the variance. Like the solution for the correct location test stimuli above, the source waveforms relating to these generators show P1 activity for the correct location targets. No activity was seen when this solution was used for the other test stimuli. Source models were produced in a step-wise fashion by fitting sources with some constraints. In most cases bilateral constraints of symmetry were enforced with anatomical constraints used to represent medial temporal contribution to the models. Attempts to construct a model without fixed sources led to dipole migration from the head model, resulting in implausible solutions.

\section{Experiment 2}

\subsection{Method}

\subsubsection{Participants}

Twenty-three participants were chosen in an ad hoc manor, all aged between 20 and 21 years ( 14 females). All participants had normal or corrected-to-normal vision and gave informed, written consent before participation. The experiment was conducted in accordance with the Code of Ethics of the World Medical Association and the ethical standards of the APA as well as abiding by the NUI Maynooth University Ethics Code.

\subsubsection{Stimuli}

The task consisted of one computer-generated environment (Grass) with 8 different objects and 2 different landmarks. Stimuli were presented as described above (see Experiment 1). 
Table 1

Dipole model information for Study and Test stimuli solutions. P1 was specifically localised as its occurrence precedes the latency differences.

\begin{tabular}{|c|c|c|c|c|c|c|}
\hline \multirow{2}{*}{$\begin{array}{l}\text { Condition } \\
\text { Study Stimuli 300-600 ms (R.V. - 2.91\%) }\end{array}$} & \multirow{2}{*}{ Dip. } & \multicolumn{3}{|c|}{ Talairach co-ordinates } & \multirow{2}{*}{$\begin{array}{l}\text { Brodmann's area } \\
\text { BA22 }\end{array}$} & \multirow{2}{*}{$\begin{array}{l}\text { Structure } \\
\text { L. Sup. Temporal Gyrus }\end{array}$} \\
\hline & & -48.2 & -35.7 & 3.1 & & \\
\hline & 2 & 48.2 & -35.7 & 3.1 & BA22 & R Sup. Temporal Gyrus \\
\hline & 3 & 22.1 & -78 & 25.6 & BA31 & R. Precuneus \\
\hline & 4 & -22.1 & -78 & 25.6 & BA31 & L. Precuneus \\
\hline & 5 & -1.6 & 44.7 & 22.4 & BA9 & L. Medial Frontal Gyrus \\
\hline & 6 & 21 & -20.1 & -5 & BA28 & R. Parahippocampal Gyrus \\
\hline & 7 & -21 & -20.1 & -5 & BA28 & L. Parahippocampal Gyrus \\
\hline \multirow[t]{7}{*}{ Test Stimuli (Correct location target) 300-600 ms (R.V. - 3.24\%) } & 1 & -51.1 & -43.9 & 9.4 & BA21 & L. Middle Temporal Gyrus \\
\hline & 2 & 51.1 & -43.9 & 9.4 & BA21 & R. Middle Temporal Gyrus \\
\hline & 3 & 18.1 & -75 & 29.3 & BA31 & R. Precuneus \\
\hline & 4 & -18.1 & -75 & 29.3 & BA31 & L. Precuneus \\
\hline & 5 & -16.5 & 40.4 & 12.2 & BA10 & L. Medial Frontal Gyrus \\
\hline & 6 & 21.3 & -19.9 & -4.7 & BA28 & R. Parahippocampal Gyrus \\
\hline & 7 & -21.3 & -19.9 & -4.7 & BA28 & L. Parahippocampal Gyrus \\
\hline \multirow[t]{7}{*}{ Test Stimuli (Incorrect location target) $300-600 \mathrm{~ms}$ (R.V. - 4.04\%) } & 1 & -52.9 & -39.7 & 10 & BA22 & L. Sup. Temporal Gyrus \\
\hline & 2 & 52.9 & -39.7 & 10 & BA22 & R Sup. Temporal Gyrus \\
\hline & 3 & 19.8 & -77.5 & 28 & BA31 & R. Precuneus \\
\hline & 4 & -19.8 & -77.5 & 28 & BA31 & L. Precuneus \\
\hline & 5 & -11.2 & 33.5 & 24.3 & BA32 & L. Anterior Cingulate Gyrus \\
\hline & 6 & 21.3 & -21 & -4.8 & BA28 & R. Parahippocampal Gyrus \\
\hline & 7 & -21.3 & -21 & -4.8 & BA28 & L. Parahippocampal Gyrus \\
\hline \multirow[t]{7}{*}{ Test Stimuli (Distractors) 300-600 ms (R.V. - 3.07\%) } & 1 & -51.1 & -43.6 & 9.3 & BA21 & L. Middle Temporal Gyrus \\
\hline & 2 & 51.1 & -43.6 & 9.3 & BA21 & R. Middle Temporal Gyrus \\
\hline & 3 & 18 & -74.9 & 29 & BA31 & R. Precuneus \\
\hline & 4 & -18 & -74.9 & 29 & BA31 & L. Precuneus \\
\hline & 5 & -16.8 & 40.4 & 12.2 & BA10 & L. Medial Frontal Gyrus \\
\hline & 6 & 22.2 & -22.1 & -4.9 & BA28 & R. Parahippocampal Gyrus \\
\hline & 7 & -22.2 & -22.1 & -4.9 & BA28 & L. Parahippocampal Gyrus \\
\hline \multirow[t]{6}{*}{ P1 localisation (Correct location target) $160-200 \mathrm{~ms}$ (R.V. - 9.61\%) } & 1 & 22.6 & -36.2 & -10.5 & BA36 & R. Parahippocampal Gyrus \\
\hline & 2 & -22.6 & -36.2 & -10.5 & BA36 & L. Parahippocampal Gyrus \\
\hline & 3 & 37.4 & 42.4 & 3.4 & BA- & R. Inferior Frontal Gyrus \\
\hline & 4 & -37.4 & 42.4 & 3.4 & BA- & L. Inferior Frontal Gyrus \\
\hline & 5 & -51.7 & -39.8 & 1.9 & BA22 & L. Middle Temporal Gyrus \\
\hline & 6 & 30.4 & -77.4 & 27.9 & BA19 & R. Cuneus \\
\hline
\end{tabular}

\subsubsection{Procedure}

The Study Block in the procedure remained unchanged (see above). The Test Block in the procedure differed by the inclusion of two new viewpoints. The test block consisted of a 12 trial practice from the studied viewpoint followed by 96 trials where participants performed the task as described above from the studied viewpoint as well as $90^{\circ}$ left of the environment and $90^{\circ}$ right of the environment. Each of the 8 study objects appeared twice in each viewpoint (once in their correct location and once in their incorrect location) and the 8 distractor objects appeared twice in each viewpoint ( 8 study objects $\times 2$ locations $\times 3$ viewpoints $)=48$ trials $+(8$ distractors $\times 3$ viewpoints $\times 2)=96$ trials. Response procedures did not change between experiments.

\subsubsection{Behavioural data analysis}

Response accuracy was calculated automatically by E-Prime $\odot$ and manually collated into accuracy totals for each response (correct, incorrect) and condition (Target Object, Target Object-Incorrect Location, Distractor condition). All latencies were also calculated automatically by E-Prime $\odot$ and grouped as described above.

\subsection{Results}

\subsubsection{Behavioural data}

Individual mean accuracy scores for each of the three stimulus conditions (target object, target object-incorrect location and distractor object condition) for each of the viewpoints (study, left, right) were collated for comparison. Overall, accuracy was high across the three conditions regardless of the viewpoint from which participants were tested. Fig. 7a (top panel) shows the percentage mean accuracy for each of these stimulus-types scored from the Study viewpoint (Correct location targets: $98.4 \pm 1.2 \%$, Incorrect location targets: $91.3 \pm 3.4 \%$, and Distractors: $98.1 \pm 0.72 \%$ ) and the novel viewpoints (Correct location targets: $95.1 \pm 1.7 \%$, Incorrect location targets: $92.4 \pm 3.2 \%$, and Distractors: $97.6 \pm 0.75 \%$ ). A repeatedmeasures ANOVA was carried out to compare the mean accuracy in the three conditions across the three viewpoints. The within-subjects comparisons revealed no main effect of stimulus-type or viewpoint but a significant interaction effect was found for stimulus $\times$ viewpoint $[F(4,88)=4.135, p<0.005]$. Subsequent Bonferroni corrected paired-samples $t$-tests found a significant difference between Correct and
Incorrect location targets for the Study viewpoint $[t(22)=3.026, p<0.05]$. The change in viewpoint to the novel view only significantly affected performance for Correct location targets, with accuracy significantly lower for the new view trials $[t(22)=3.761, p<0.01]$. When paired-samples comparisons were made between stimulus-types regardless of viewpoint, accuracy was found to be significantly diminished for the Incorrect location condition compared to Correct location $[t(68)=3.187, p<0.05]$ and Distractor stimuli $[t(68)=2.918, p<0.05]-$ Bonferroni corrected (see Fig. 7a, bottom panel).

Reaction times (from accurate trials) are shown in Fig. 7b (top panel) and can be seen to be quicker on average for Correct location targets $(653.43 \pm 22.86 \mathrm{~ms}$ ) compared to Incorrect location targets $(721.91 \pm 22.43 \mathrm{~ms})$ and Distractors $(715.72 \pm 13.71 \mathrm{~ms})$ from the Study viewpoint and Correct location targets $(702.19 \pm 24.26 \mathrm{~ms})$ compared to Incorrect location targets $(744.44 \pm 31.72 \mathrm{~ms})$ but not Distractors ( $694.15 \pm 15.14 \mathrm{~ms}$ ) from the novel viewpoints. Fig. $7 \mathrm{~b}$ (bottom panel) shows mean reaction time grouped by stimulus-type (Correct location targets $685.94 \pm 14.51 \mathrm{~ms}$; Incorrect location targets $736.93 \pm 17.51 \mathrm{~ms}$; and Distractors $701.34 \pm 8.94 \mathrm{~ms}$ ). A repeated-measures ANOVA revealed that reaction times differed significantly with stimulus-type $[F(2,44)=4.821, p<0.05]$ but no main effect was found for viewpoint. An interaction effect of stimulus $\times$ viewpoint was found $[F(4$, $88)=9.423, p<0.001]$. Bonferroni corrected $t$-tests comparing reaction times in trials from the Study view found significant differences between Correct location targets and Incorrect location targets $[t(22)=4.356, p<0.01]$ and Correct location targets and Distractors $[t(22)=3.398, p<0.05]$. Participants were also found to respond significantly faster on the novel view trials when the target object was correctly vs. incorrectly positioned $[t(22)=2.895, p<0.05]$. Participants' reaction times were slower, on average, for the novel viewpoints compared with the study viewpoint, significantly so for Correct location targets $[t(22)=2.657, p<0.05]$ and Distractors $[t(22)=2.810, p<0.01]$ but not Incorrect location targets. Comparing stimulus-type regardless of view (Fig. $7 \mathrm{~b}$, bottom panel) revealed a significant difference between Correct and Incorrect location targets $[t(68)=4.858, p<0.01]$ - Bonferroni corrected.

\section{Discussion}

Behaviourally, it was expected that response accuracy would be greater and response latency would be faster upon presentation of 
(a)

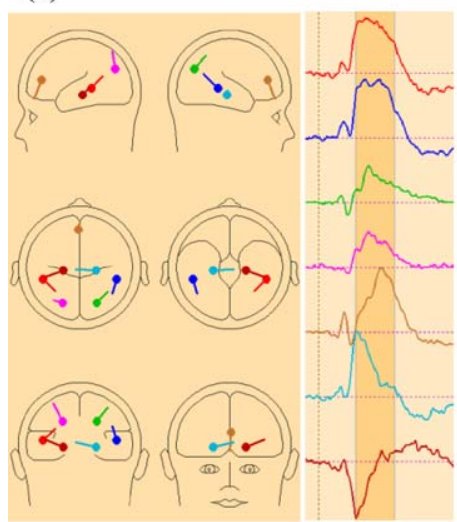

(b)

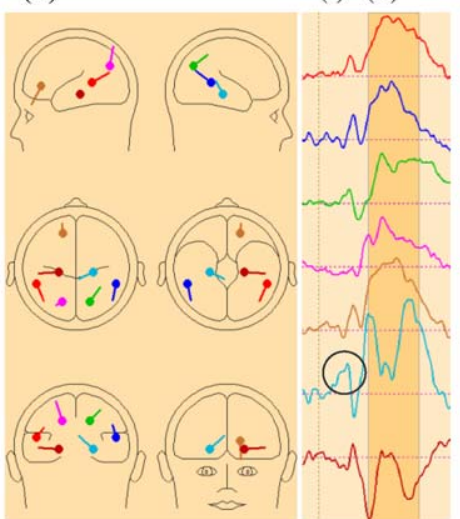

(i) (ii)

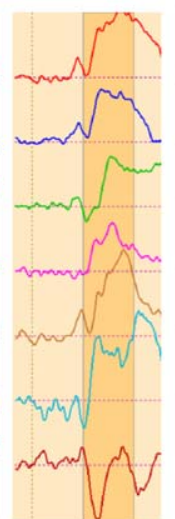

(iii)

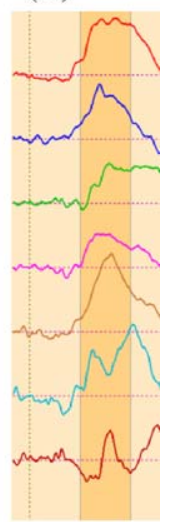

(c)
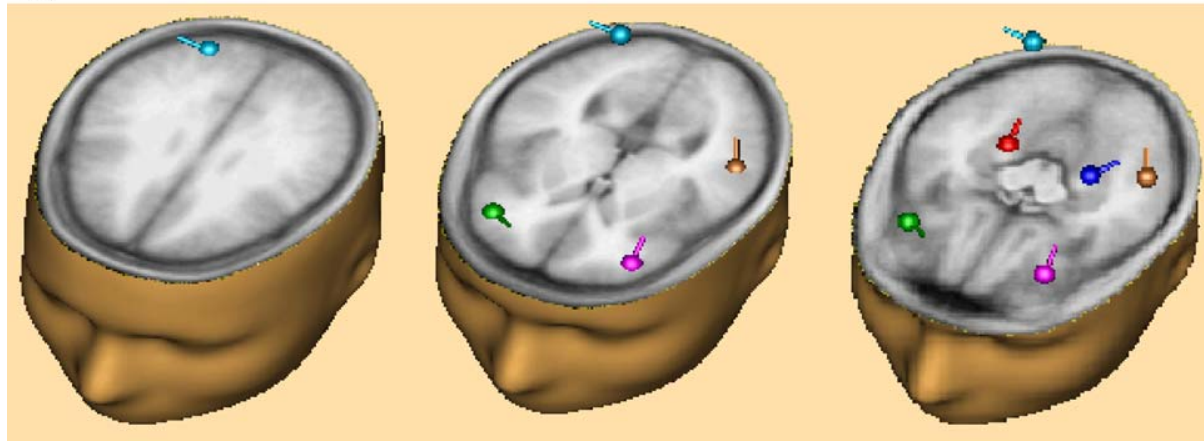

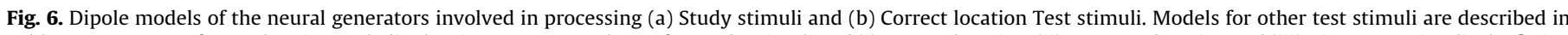

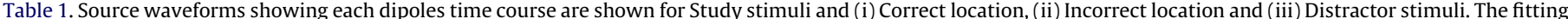

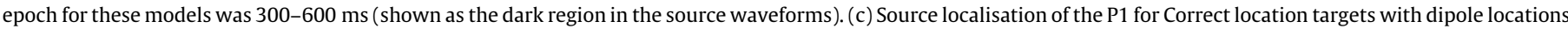

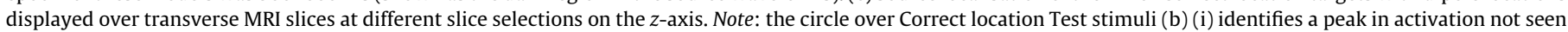
for Incorrect location (ii) or Distractor (iii) stimuli. This 'P1' is most prominent for the right parahippocampal source but can be seen for all sources in the model.

studied or target objects in their correct location than target objects in an incorrect location. The pattern of accuracy data found was similar to item-context association tasks (Rugg et al., 1998) where accuracy was greater for target objects in their correct location than those placed in an incorrect location. Accuracy data from Experiment 1 were found to differ significantly after an ANOVA was performed but subsequent non-significant $t$-tests provided no clarification. Reaction times differed significantly as predicted with correct targets being identified the fastest. Experiment 2 was undertaken to allay criticisms that the effects observed in both the behavioural and the ERP data from experiment one were due to simple scene recognition. Specifically, trials where the test object appeared in its correct or studied location would be recognized as a scene compared to trials in which object or object-location had changed from the study phase. On review of the behavioural data from both experiments it can be argued that although scene recognition may play a role, the effect of viewpoint on memory differs from the effect of stimulus location. The viewpoint changes in experiment two lead to some decreases in overall performance but the pattern of performance across stimulus-types was similar in the old and new viewpoints. In addition, reaction times differed significantly with stimulustype but no main effect was found for viewpoint. In both experiments there was no difference in accuracy scores between targets and distractors, suggesting that the participants clearly understood the instructions of the task. The reaction time data are a more useful measure of whether implicit learning occurred and based on the data reported there is a strong argument this was the case. Indeed after the median split, in an alternative analysis, reaction times were shown to differ significantly for the object recognition task but these differences were nullified when the object was presented in its original location. Among the characteristic differences between explicit and implicit memory, outlined by Reber (1993), is the low variability (individual differences) in implicit learning and memory compared to explicit learning and memory. The variance between the 'Good Recognition Group' and 'Poor Recognition Group' seemed to be dependant on whether the objects' spatial location was (re)presented. This variance was also seen in the ERP findings where amplitude differences observed between the groups were not present when objects appeared in their correct locations. The results from ERP and source data will be discussed separately at first in subsections relating to stimulus processing and stimulus-type. A more general discussion will follow including an elaboration on the sex differences identified.

\subsection{Encoding}

The processing of the Study stimuli elicited a P300 identified over parietal scalp electrodes. The P300 component is thought to be composed of several parts that reflect an information processing cascade when attentional and memory mechanisms are engaged (Polich, 2007). The model described by Polich (2007) posits that "the P300" comprises an early attention process stemming from a frontal working memory representational change to produce the P3a. The attention-driven stimulus signal is then transmitted to temporal and parietal structures related to P3b. Dipole source analysis revealed a distributed network involving frontal, parietal, temporal, and medial temporal sources. With an $\mathrm{RV}$ of $<3 \%$, the model generated to account for the scalp pattern recorded for 

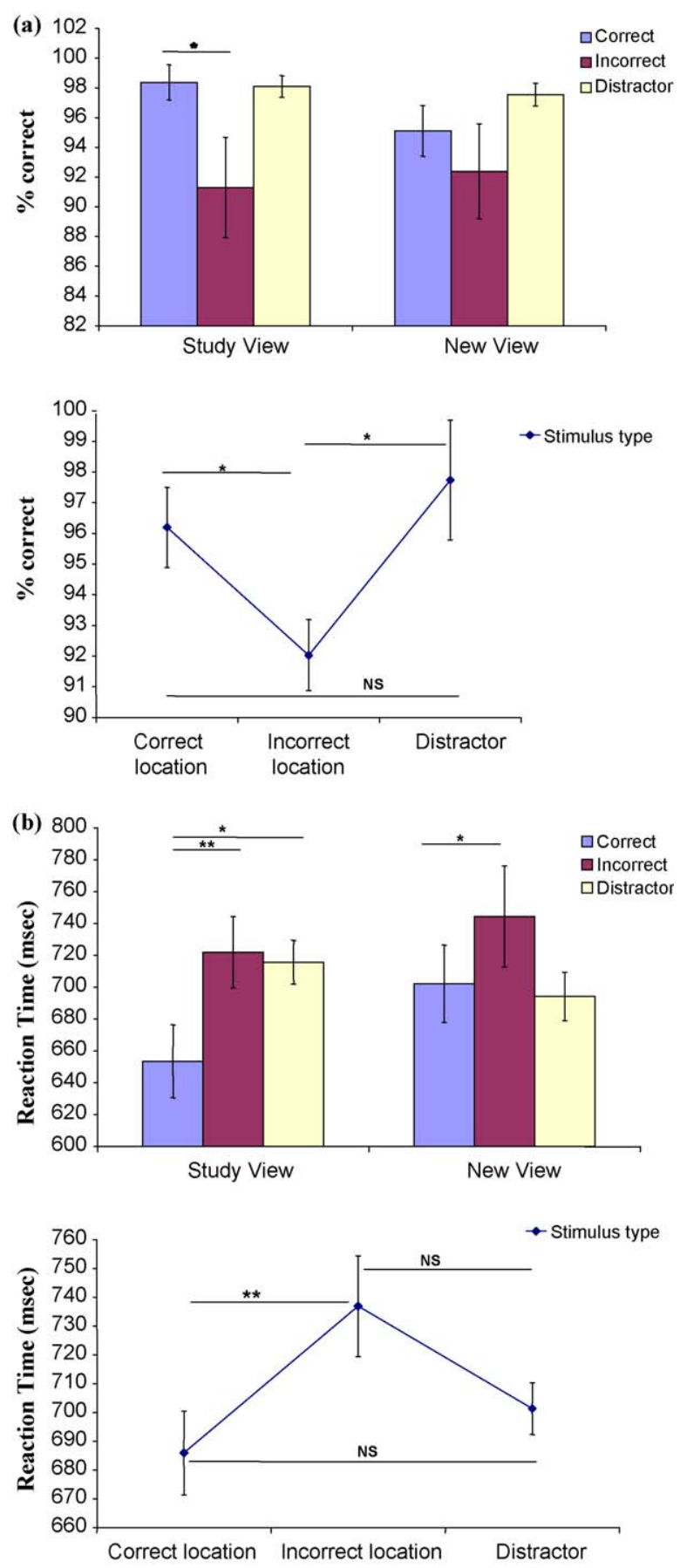

Fig. 7. (a) Mean accuracy for each of the stimulus-type conditions for the different viewpoints tested (left and right views collated into 'new' view condition). Mean accuracy for each of the stimulus-type conditions regardless of viewpoint is also displayed. Error bars display Standard Error of the Mean. (b) Mean reaction times for each of the stimulus-type conditions for the different viewpoints tested (left and right views collated into 'new' view condition). Mean reaction times for each of the stimulus-type conditions regardless of viewpoint are also shown. Error bars display Standard Error of the Mean. ${ }^{*} p<0.05,{ }^{* *} p<0.01$, NS $=$ not significant.

Study stimuli includes regions that may be involved in processing verbal attributes of the objects (frontotemporal) as well as spatial aspects of the environment (medial temporal and parietal).

\subsection{Retrieval}

Differences between the ERP waveforms elicited by Study and Test stimuli could be seen in the extended duration of P300 positivity for Test stimuli most likely indicating additional stimulus evaluation processing and response selection. The processing of the Test stimuli led to a latency difference in the elicitation of a parietal P300, dependant on object-location variance from the encoding phase. P300 latency is thought to index classification speed, which is proportional to the time required to detect and evaluate a target stimulus (Kutas et al., 1977; Magliero et al., 1984). The latency difference was significant, where earlier positive-going fluctuations were recorded for Correct location Targets. So in addition to the reaction time data, participants physiologically classified test objects more quickly when they were presented in their studied location. Source analysis showed the same (or similar) neural generators were involved in retrieval of test objects regardless of stimulus-type. Therefore the latency difference reveals an earlier activation of an underlying process or network which recruits the same areas. These areas, namely frontal (BA10, 32) and temporal gyri (BA21, 22 ), the precuneus (BA31) and parahippocampus (BA28) were also active for the encoding of the Study stimuli.

BA28 denotes a specific area of the parahippocampus, the entonrhinal cortex (EC). The EC provides the critical input pathway in this area of the brain, linking the association cortices to the hippocampus. It also provides the pathway for signals returning from the hippocampus to the association cortices. Due to its role as a major convergence zone, both for object information arriving from perirhinal cortex and spatial information arriving from parahippocampal cortex, its function in object-location memory has been tested in numerous studies in rats (Parron and Save, 2004; Parron et al., 2006), primates (Insausti et al., 1987; Suzuki and Amaral, 1994; Suzuki et al., 1997) and humans (Haist et al., 2001; Miller et al., 1998).

BA31, which is positioned between the cingulate and splenial sulci, includes both posterior cingulate and precuneate cortices. The precuneus has been implicated in spatial attention (Le et al., 1998), allocentric spatial memory (Frings et al., 2006), mental navigation (Ghaem et al., 1997), real-world navigation (Suzuki et al., 1998) and visual imagery in episodic memory recall (Buckner et al., 1995; Fletcher et al., 1996; Halsband et al., 1998). The principal extraparietal corticocortical connections of the precuneus are with the frontal lobes (Cavanna and Trimble, 2006). The precuneus has reciprocal projections to the prefrontal cortex as well as superior temporal sulci, areas also included in the dipole models.

Frontal regions, such as mid-prefrontal area (BA10) and anterior cingulate cortex (ACC-BA32) are posited to be involved in a cognitive control network for processing context and monitoring performance (Gutchess et al., 2007; Ridderinkhof et al., 2004) and working memory in general. The shift in frontal activations to the ACC during Incorrect location Target processing may indicate a task-difficulty increase or an increase in self-monitoring or error processing. Behaviourally most errors occurred for these stimuli. Activation of the ACC has recently been shown for errors made with and without awareness (Hester et al., 2005).

Temporal activations were more superior than expected for object processing. Dipoles were located bilaterally either in middle or superior temporal gyri (BA21/22). The inferior temporal gyrus is one of the higher levels of the ventral stream of visual processing and there are an extensive number of studies highlighting this areas role in object representation (Gross, 1994; Wachsmuth et al., 1994). However, Duzel et al. (1999) conducted a PET and ERP study to distinguish between the neural correlates of task-related (episodic/semantic) and item-related (old/new) processes of memory retrieval. They reported temporal lobe activation in BA21 for the semantic retrieval task. This co-occurred with left frontal activations similar to those found in the current study. The easily accessible semantic properties of the unambiguous objects 
used in the current study may have been responsible for the unpredicted temporal lobe activations.

As mentioned above, processing of test stimuli recruited very similar brain areas leaving the latency difference to be explained. Although significance was not reached in the comparison of P1 amplitudes across stimulus-types, it is posited that the earlier activation which occurred for Correct location Targets was associated with a P1, specifically related to same-location facilitation. This component can be seen in the source waveforms relating to the dipole models but is only evident for the model pertaining to Correct location Targets. In particular, activation of the right parahippocampal gyrus can be seen to occur when the test object was presented in its 'correct' location (Fig. 6b(i)). Localising the P1 for Correct location Targets separately, bilateral sources were found in BA36 (parahippocampal cortex), the area that relates spatial information on to the EC. Frontal and temporal generators were again identified. A source in the cuneus suggests processing earlier in the visual system before the parietal processing documented in the precuneus. When this model was applied to the other test stimuli over the same fitting interval $(160-200 \mathrm{~ms})$, the RV was $50+\%$, a further indication of the importance of these activations for the latency effect to occur dependant on implicit location memory. Activations of the hippocampus would suggest the involvement of explicit memory. The MTL dipoles contradict previous research which has shown a differentiation in terms of brain areas involved in explicit and implicit memory, with activations of the medial temporal lobes for explicit and the basal ganglia for implicit (Honda et al., 1998; Poldrack et al., 2001). However, some argue that MTL pathology produces implicit memory impairment that often goes undetected (Ostergaard and Jernigan, 1993; Ostergaard, 1999; Jernigan et al., 2001) and a number of functional magnetic resonance studies have reported medial temporal lobe activation in implicit learning (see for review, Forkstam and Petersson, 2005).

The amplitude differences recorded between males and females indicate a sex difference, possibly related to differential strategy implementation, which shows additional left hemispheric cortical activation for females over males. Data from Alexander et al. (2002) suggest that the processing of object features and object identification in the left cerebral hemisphere may include processing of spatial information that may contribute to superior object-location memory in females relative to males. The female superiority for object-location memory was first explored by Silverman and Eals (1992) who developed a task intended to measure object-location memory and reported that females outperformed males on their paper and pencil version of this task. This has been confirmed by other research (James and Kimura, 1997). Our data extend previous claims for sex differences in object-location memory by demonstrating electrophysiological sex differences consistent with an evolutionary model (Eals and Silverman, 1994; Silverman et al., 2007).

We demonstrated, both behaviourally and physiologically, that humans can classify objects more quickly when using implicit spatial memory. As the task was an object recognition task with reaction time being an explicit measure of performance, the P300 amplitudes may represent task proficiency, as mean amplitudes correlated significantly with reaction time. Differences observed in object recognition proficiency were absent if objects were presented in their correct location. Correct location presentations implicitly aided object recognition as they removed both behavioural and electrophysiological performance differences. A network of structures were identified in frontal, parietal and temporal areas with subdivisions of the parahippocampus playing a crucial role in the implicit recognition of location and its facilitation of concurrent explicit object recognition. In conclusion, we propose an electrophysiologically based model of implicit spatial memory which demonstrates the influence of objectlocation memory on P300 latency and amplitude and the importance of this component as well as earlier spatially related components for object recognition.

\section{Acknowledgment}

This work was funded by the Irish Research Council for Science, Engineering and Technology.

\section{References}

Aggleton, J.P., Brown, M.W., 1999. Episodic memory amnesia and the hippocampalanterior thalamic axis. Behavioral and Brain Sciences 22, 425-444.

Alexander, G.M., Packard, M.G., Peterson, B.S., 2002. Sex and spatial position effects on object location memory following intentional learning of object identities. Neuropsychologia 40, 1516-1522.

Andersen, R.A., Essick, G.K., Siegel, R.M., 1985. Encoding of spatial location by posterior parietal neurons. Science 230 (4724), 456-458.

Baddeley, A.D., 1990. Human Memory: Theory and Practice. .

Berg, P., Scherg, M., 1991. Dipole models of eye movements and blinks. Electroencephalography \& Clinical Neurophysiology 79, 36-44.

Bowers, C.A., Martin, L., Price, C., 1998. Dual-task performance and the lateralization of spatial orientation: artifact of test selection? Journal of General Psychology $125,5-16$.

Buckner, R.L., Peterson, S.E., Ojemann, J.G., Miezin, F.M., Squire, L.R., Raichle, M.E. 1995. Functional anatomical studies of explicit and implicit memory retrieval tasks. Journal of Neuroscience 15, 12-29.

Caldwell, J.I., Masson, M.E., 2001. Conscious and unconscious influences of memory for object location. Memory \& Cognition 29, 285-295.

Cavanna, A.E., Trimble, M.R., 2006. The precuneus: a review of its functional anatomy and behavioural correlates. Brain 129 (3), 564-583.

Cherney, I.D., Ryalls, B.I., 1999. Gender-linked differences in incidental memory of children and adults. Journal of Experimental Child Psychology 72, 305-328.

Chun, M.M., 2000. Contextual cueing of visual attention. Trends in Cognitive Sciences 4, 170-178.

Chun, M.M., Jiang, Y., 1998. Contextual cueing: implicit learning and memory of visual context guides spatial attention. Cognitive Psychology 36, 28-71.

Chun, M.M., Marois, R., 2002. The dark side of visual attention. Current Opinion in Neurobiology $12,184-189$.

Commins, S., Cunningham, L., Harvey, D., Walsh D, 2003. Massed but not spaced training impairs spatial memory. Behavioural Brain Research 139, 215-223.

Crane, J., Milner, B., Leonard, G., 1995. Spatial-array learning by patients with focal temporal-lobe excisions. Society for Neuroscience Abstracts 21, 1446.

Duzel, E., Cabeza, R., Picton, T.W., Yonelinas, A.P., Scheichi, H., Heinze, H., Tulving E, J., 1999. Task-related and item-related brain processes of memory retrieval. Proceedings of the National Academy of Sciences of the United States of America 96, 1794-1799.

Eals, M., Silverman, I., 1994. The hunter-gatherer theory of spatial sex differences: proximate factors mediating the female advantage in recall of object arrays Ethology and Sociobiology 15, 95-105.

Ennaceur, A., Neave, N., Aggleton, J.P., 1997. The effects of neurotoxic lesions of the perirhinal cortex combined to fornix transection on object recognition memory in the rat. Behavioral Brain Research 88, 181-193.

Fletcher, P.C., Shallice, T., Frith, C.D., Frackowiak, R.S., Dolan, R.J., 1996. Brain activity during memory retrieval: the influence of imagery and semantic cueing. Brain 119, 1587-1596.

Forkstam, C., Petersson, K.M., 2005. Towards an explicit account of implicit learning. Current Opinion in Neurology 18, 435-441.

Frings, L., Wagner, K., Quiske, A., Schwarzwald, R., Spreer, J., Halsband, U., SchulzeBonhage, A., 2006. Precuneus is involved in allocentric spatial location encoding and recognition. Experimental Brain Research 173 (4), 661-672.

Galani, R., Weiss, I., Cassel, J.C., Kelche, C., 1998. Spatial memory, habituation, and reactions to spatial and nonspatial changes in rats with selective lesions of the hippocampus, the entorhinal cortex or the subiculum. Behavioral Brain Research 96, 1-12.

Ghaem, O., Mellet, O., Crivello, F., Tzourio, N., Mazoyer, B., Berthoz, A., Denis, M., 1997. Mental navigation along memorized routes activates the hippocampus, precuneus and insula. NeuroReport 8, 739-744.

Goldman-Rakic, P., 1987. Circuitry of primate prefrontal cortex and regulation of behavior by representational memory. Handbook of Physiology 5 (9), 373-417

Gross, C.G., 1994. How inferior temporal cortex became a visual area. Cerebral Cortex 4, 455-469.

Gutchess, A.H., Hebrank, A., Sutton, B., Leshikar, E., Chee, M.W.L., Tan, J.C., Goh, J.O.S., Park, D.C., 2007. Contextual interference in recognition memory with age. NeuroImage $35,1338-1347$.

Haist, F., Bowden, G.J., Mao, H., 2001. Consolidation of human memory over decades revealed by functional magnetic resonance imaging. Nature Neuroscience 4 1139-1145.

Halsband, U., Krause, B.J., Schmidt, D., Herzog, H., Tellmann, L., Müller-Gärtner, H.W., 1998. Encoding and retrieval in declarative learning: a positron emission tomography study. Behavioral Brain Research 97, 69-78. 
Hasher, L., Zacks, R.T., 1979. Automatic and effortful processes in memory. Journal of Experimental Psychology 108 (3), 356-388.

Hay, J.F., Jacoby, L.L., 1996. Separating habit and recollection: memory slips, process dissociations, and probability matching. Journal of Experimental Psychology: Learning, Memory, \& Cognition 22, 1323-1335.

Hester, R., Shpaner, M., Molholm, S., Foxe, J.J., Garavan, H., 2005. Neural correlates of error detection with and without awareness. NeuroImage 27, 602-608.

Honda, M., Deiber, M.P., Ibanez, V., Pascual-Leone, A., Zhuang, P., Hallett, M., 1998 Dynamic cortical involvement in implicit and explicit motor sequence learning. A PET study. Brain 121, 2159-2173.

Ille, N., Berg, P., Scherg, M., 2002. Artifact correction of the ongoing EEG using spatial filters based on artifact and brain signal topographies. Journal of Clinica Neurophysiology 19, 113-124.

Insausti, R., Amaral, D.G., Cowan WM, 1987. The entorhinal cortex of the monkey. II. Cortical afferents. Journal of Comparative Neurology 264, 356-395.

Jacoby, L.L., 1991. A process dissociation framework: separating automatic from intentional uses of memory. Journal of Memory \& Language 30, 513-541.

Jacoby, L.L., Kelley, C.M., 1992. A process-dissociation framework for investigating unconscious influences: freudian slips, projective tests, subliminal perception and signal detection theory. Current Directions in Psychological Science 1 (6), $174-179$.

James, T.W., Kimura, D., 1997. Sex differences in remembering the locations of objects in an array: location-shifts versus location-exchanges. Evolution and Human Behavior 18, 155-163.

Jernigan, T.L., Ostergaard, A.L., Fennema-Notestine, C., 2001. Mesial temporal, diencephalic, and striatal contributions to deficits in single word reading, word priming, and recognition memory. Journal of the International Neuropsychological Society 7, 63-78.

Johnsrude, I.S., Owen, A.M., Crane, J., Milner, B., Evans, A.C., 1999. A cognitive activation study of memory for spatial relationships. Neuropschologia 37 829-841.

Kosslyn, S.M., Chabris, C.F., Marsolek, C.J., Koenig, O., 1992. Categorical versus coordinate spatial relations: computational analyses and computer simulation. Journal of Experimental Psychology: Human Perception and Performance 18 562-577.

Kutas, M., McCarthy, G., Donchin, E., 1977. Augumenting mental chronometry: the P3 as a measure of stimulus evaluation time. Science 197, 792-795.

Lancaster, J.L., Summerln, J.L., Rainey, L., Freitas, C.S., Fox PT, 1997. The Talairach Daemon, a database server for Talairach Atlas Labels. Neuroimage 5, S633.

Le, T.H., Pardo, J.V., Hu X, 1998. 4 T-fMRI study of nonspatial shifting of selective attention: cerebellar and parietal contributions. Journal of Neurophysiology 79 , 1535-1548.

Magliero, A., Bashore, T.R., Coles, M.G., Donchin, E., 1984. On the dependence of P300 latency on stimulus evaluation processes. Psychophysiology 21, 171-186.

Mecklinger, A., Opitz, B., Friederici, A.D., 1997. Semantic aspects of novelty detection in humans. Neuroscience Letters 234, 1-4

Miller, L.A., Lai, R., Munoz, D.G., 1998. Contributions of the entorhinal cortex amygdala and hippocampus to human memory. Neuropsychologia 36, 12471256.

Milner, B., Johnsrude, I., Crane, J., 1997. Right medial temporal-lobe contribution to object-location memory. Philosophical Transactions of the Royal Society of London Series B 352, 1469-1474.

Molholm, S., Ritter, W., Javitt, D.C., Foxe, J.J., 2004. Multisensory visual-auditory object recognition in humans: a high-density electrical mapping study. Cerebra Cortex 14, 452-465.

Mumby, D.G., Pinel, J.P.J., 1994. Rhinal cortex lesions and object recognition in rats. Behavioral Neurology 108, 11-18.

Olson, I.R., Chun, M.M., 2002. Perceptual constraints on implicit learning of spatial context. Visual Cognition 9, 273-301.

Ostergaard, A.L., Jernigan, T.L., 1993. In: Graf, P., Masson, M.E.J. (Eds.), Are Word Priming and Explicit Memory Mediated by Different Brain Structures? Implicit Memory. Erlbaum, Hillsdale, NJ, pp. 327-349.

Ostergaard, A.L., 1999. Priming deficits in amnesia: now you see them, now you don't. Journal of the International Neuropsychological Society. 5, 175190.

Owen, A.M., Milner, B., Petrides, M., Evans, A.C., 1996. A specific role for the right parahippocampal gyrus in the retrieval of object-location: a positron emission tomography study. Journal of Cognitive Neuroscience 8, 588-602.

Parron, C., Save, E., 2004. Comparison of the effects of entorhinal and retrosplenial cortical lesions on habituation, reaction to spatial and non-spatial changes during object exploration in the rat. Neurobiology of Learning \& Memory 82, $1-11$.
Parron, C., Poucet, B., Save, E., 2006. Cooperation between the hippocampus and the entorhinal cortex in spatial memory: a disconnection study. Behavioural Brain Research 170, 99-109.

Poldrack, R.A., Clark, J., Pare-Blagoev, E.J., Shohamy, D., Creso Moyano, J., Myers, C., Gluck, M.A., 2001. Interactive memory systems in the human brain. Nature 414, $546-550$.

Polich, J., 2007. Updating P300: an integrative theory of P3a and P3b. Clinical Neurophysiology $118,2128-2148$.

Postma, A., De Haan, E.H.F., 1996. What was where? Memory for object locations. Quarterly Journal of Experimental Psychology 49A, 178-199.

Puglisi, J.H., Park, D.C., Smith, A.D., Hill, G.W., 1985. Memory for two types of spatial location: effects of instructions, age, and format. The American Journal of Psychology 98 (1)

Reber, A.S., 1993. Implicit Learning and Tacit Knowledge: An Essay on the Cognitive Unconscious. Oxford University Press, NY.

Richardson, D.C., Spivey, M.J., 2000. Representation, space and Hollywood Squares: looking at things that aren't there anymore. Cognition 76, 269-295.

Ridderinkhof, K.R., Ullsperger, M., Crone, E.A., Nieuwenhuis, S., 2004. The role of the medial frontal cortex in cognitive control. Science 306, 443-447.

Roche, R.A.P., Mangaoang, M.A., Commins, S., O’Mara, S.M., 2005. Hippocampal contributions to neurocognitive mapping in humans: a new model. Hippocampus $15,622-641$.

Rugg, M.D., Schloerscheidt, A.M., Mark, R.E., 1998. An electrophysiological comparison of two indices of recollection. Journal of Memory and Language 39, 47-69.

Ryan, J.D., Althoff, R.R., Whitlow, S., Cohen NJ, 2000. Amnesia is a deficit in relational memory. Psychological Science 11, 454-461.

Save, E., Poucet, B., Foreman, N., Buhot, N., 1992. Object exploration and reactions to spatial and nonspatial changes in hooded rats following damage to parietal cortex or hippocampal formation. Behavioral Neuroscience 106, 447-456.

Scherg, M., Picton, T.W., 1991. Separation and identification of event-related potential components by brain electric source analysis. Electroencephalography and Clinical Neurophysiology Supplemental 42, 24-37.

Sehatpour, P., Molholm, S., Javitt, D.C., Foxe, J.J., 2006. Spatiotemporal dynamics of human object recognition processing: an integrated high-density electrical mapping and functional imaging study of "closure" processes. Neuroimage 29, 518-605.

Silverman, I., Eals, M., 1992. Sex differences in spatial abilities: evolutionary theory and data. In: Barkow, J., Cosmides, L., Tooby, J. (Eds.), Evolutionary Psychology and the Generation of Culture. Oxford University Press, New York, pp. 487-503.

Silverman, I., Choi, J., Peters, M., 2007. The hunter-gatherer theory of sex differences in spatial abilities: data from 40 countries. Archives of Sexual Behavior 36, $261-$ 268.

Smith, M.L., Milner, B., 1981. The role of the right hippocampus in the recall of spatial location. Neuropsychologia 19, 781-793.

Smith, M.L., Milner, B., 1984. Differential effects of frontal lobe lesions on cognitive estimation and spatial memory. Neuropsychologia 22, 697-705.

Smith, M.L., Milner, B., 1989. Right hippocampal impairment in the recall of spatial location: encoding deficit or rapid forgetting? Neuropsychologia 27, 71-81.

Steckler, T., Sahgal, A., Aggleton, J.P., Drinkenburg, W.H.I.M., 1998. Recognition memory in rats. III. Neurochemical substrates. Progress in Neurobiology 54 333-348.

Suzuki, W.A., Amaral, D.G., 1994. Topographic organization of the reciprocal connections between the monkey entorhinal cortex and the perirhinal and parahippocampal cortices. Journal of Neuroscience 14, 1856-1877.

Suzuki, W.A., Miller, E.K., Desimone, R, 1997. Object and place memory in the macaque entorhinal cortex. Journal of Neurophysiology 78, 1062-1081.

Suzuki, K., Yamadori, A., Hayakawa, Y., Fujii, T., 1998. Pure topographical disorientation related to dysfunction of the viewpoint dependent visual system. Cortex 34, 589-599.

Turner, J., 1993. Incidental information processing: effects of mood, sex and caffeine. International Journal of Neuroscience 72, 1-14

Ullman, S., 1996. High-level Vision: Object Recognition and Visual Cognition. MIT Press, Cambridge, MA

Van Asselen, M., Fritschy, E., Postma, A., 2005. The influence of intentional and incidental learning on acquiring spatial knowledge during navigation. Psychological Research 70, 151-156.

Wachsmuth, E., Oram, M., Perrett, D., 1994. Recognition of objects and their component parts: responses of single units in the temporal cortex of the macaque. Cerebral Cortex 4 (5), 509-522.

Wang, H., Johnson, T.R., Zhang, J., Wang, Y., 2002. A study of object-location memory. In: Proceedings of the Twenty-Fourth Annual Conference of the Cognitive Science Society, Lawrence Erlbaum, Hillsdale, NJ. 